\title{
Complex-Clifford Tori and Special Complex Unitary Matrices
}

\section{Hasan $\mathrm{ES}^{1^{*}}$}

ABSTRACT: In this paper, parallels of latitude and meridians of longitude in $\boldsymbol{S}_{\mathbb{C}}^{3}$ are identified via the special complex unitary matrices $\boldsymbol{S U}_{\mathbb{C}}(2)$. It is also obtained that the third homology group of complex 2-sphere $\boldsymbol{S}_{\mathbb{C}}^{2}$ is equal to zero.

Keywords: Real quaternion algebra; complexified quaternion algebra; complex-clifford tori.

${ }^{1}$ Hasan ES (Orcid ID: 0000-0002-7732-8173), Gazi Üniversitesi, Gazi Eğitim Fakültesi, Matematik ve Fenbilimleri Eğitimi Bölümü, Ankara, Türkiye

*Sorumlu Yazar/Corresponding Author: Hasan Es, e-mail: hasanes@ gazi.edu.tr 


\section{INTRODUCTION}

Unit real quaternions groups of $\boldsymbol{S}^{3}$ and unitary matrices $\boldsymbol{S} \boldsymbol{U}(2)$ are isomorphic (Chevalley, 1946). Therefore, parallels of latitude and meridians of longitude in $\boldsymbol{S}^{3}$ can be given by the elements of $\boldsymbol{S U}(2)$. Moreover, special orthogonal matrices $\boldsymbol{S O}\left(I R^{3}\right)$ correspond to $\boldsymbol{S}^{3}$. Also, $\boldsymbol{S O}\left(I R^{3}\right)$ is isomorphic to 3dimensional Euclidean projective space $I R P^{3}$. Third homology group of $\boldsymbol{S}^{2}$ equals to zero based on Hopf fibriation (Toth, 1998).

In this paper, unit complexified quaternions $\boldsymbol{S}_{\mathbb{C}}^{3}$ are constructed by bicomplex numbers $\mathbb{C}_{2}$. Additionally, parallels of latitude and meridians of longitude in $\boldsymbol{S}_{\mathbb{C}}^{3}$ are determined based on special complex unitary matrices $\boldsymbol{S} \boldsymbol{U}_{\mathbb{C}}(2)$. Also, it is shown that special complex orthogonal matrices $\boldsymbol{S O}\left(\mathbb{C}^{3}\right)$ are isomorphic to 3-dimensional complex projective space $\mathbb{C} P^{3}$ using Hopf fibriation, it is revealed that third homology group of complex 2-sphere $\boldsymbol{S}_{\mathbb{C}}^{2}$ is zero.

In particular, some known relations about the concepts, which are mentioned above, for the real quaternions are found by assuming quaternion imaginary components of complexified quaternions' equal to zero.

\section{MATERIALS AND METHODS}

\section{Real Quaternion Algebra}

Real quaternion algebra $\mathcal{H}$ is an associative, non-commutative division ring with basis elements $1, \boldsymbol{i}, \boldsymbol{j}, \boldsymbol{k}$ obeying the following multiplication rules (Hamilton, 1844):

$i^{2}=j^{2}=k^{2}=i j k=-1$ and $i j=-j i=k, j k=-k j=i, k i=-i k=j$.

Real quaternions are classically shown in the hypercomplex number forms with three imaginary components. This is $q=q_{0}+q_{1} \boldsymbol{i}+q_{2} \boldsymbol{j}+q_{3} \boldsymbol{k}$ for a real quaternion $q$ in Cartesian form, where $q_{0}, q_{1}, q_{2}, q_{3}$ are real numbers. If $q_{0}=0$ then $q$ is regarded as pure real quaternion. Pure real quaternions form the 3-dimensional linear subspace $\operatorname{Im} \mathcal{H}=\left\{q_{1} \boldsymbol{i}+q_{2} \boldsymbol{j}+q_{3} \boldsymbol{k}, q_{1}, q_{2}, q_{3} \in I R\right\}$ of $\mathcal{H}$. The conjugate of $q$ is $\bar{q}=q_{0}-q_{1} \boldsymbol{i}-q_{2} \boldsymbol{j}-q_{3} \boldsymbol{k}$. For $q \in \operatorname{Im} \mathcal{H}, \bar{q}$ is equal to $q$.

A real quaternion $q=q_{0}+q_{1} \boldsymbol{i}+q_{2} \boldsymbol{j}+q_{3} \boldsymbol{k}$ can be given in different forms. Three of them are shown below:

1) $q=S(q)+\boldsymbol{V}(q)$ where $S(q)=q_{0}$ is the scalar part and $\boldsymbol{V}(q)=q_{1} \boldsymbol{i}+q_{2} \boldsymbol{j}+q_{3} \boldsymbol{k}$ is the vector part of $q$. In this form $\bar{q}=S(q)-\boldsymbol{V}(q)$.

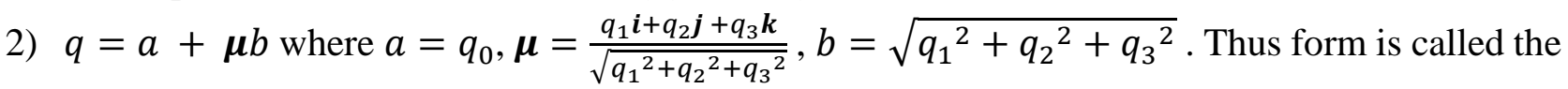
complex form of $q$, and $\bar{q}=a-\boldsymbol{\mu} b$.

3) $q=\sqrt{N_{q}}(\cos \varphi+\widehat{\boldsymbol{q}} \sin \varphi)$, where $N_{q}=\|q\|=q_{0}{ }^{2}+q_{1}{ }^{2}+q_{2}{ }^{2}+q_{3}{ }^{2}, \quad \cos \varphi=q_{0} / \sqrt{N_{q}}$, $\sin \varphi=\sqrt{{q_{1}}^{2}+q_{2}{ }^{2}+q_{3}{ }^{2}} / \sqrt{N_{q}}, \quad \hat{\boldsymbol{q}}=\left(q_{1} \boldsymbol{i}+q_{2} \boldsymbol{j}+q_{3} \boldsymbol{k}\right) / \sqrt{q_{1}{ }^{2}+q_{2}{ }^{2}+q_{3}{ }^{2}}$. The from is called the polar form, and $\bar{q}=\sqrt{N_{q}}(\cos \varphi-\widehat{q} \sin \varphi)$.

Summation of the real quaternions $q$ and $p$ is $q+p=(S(q)+S(p))+(\boldsymbol{V}(q)+\boldsymbol{V}(p))$. Multiplication a real quaternion $q$ with a scalar $\lambda$ is $\lambda q=\lambda S(q)+\lambda \boldsymbol{V}(q)$. Multiplication of real quaternions $q$ and $p$ is

$q p=S(q) S(p)-\langle\boldsymbol{V}(q), \boldsymbol{V}(p)\rangle+S(q) \boldsymbol{V}(p)+S(p) \boldsymbol{V}(q)+\boldsymbol{V}(q) \wedge \boldsymbol{V}(p)$,

where

$$
\langle\boldsymbol{V}(q), \boldsymbol{V}(p)\rangle=q_{1} p_{1}+q_{2} p_{2}+q_{3} p_{3}
$$

and

$$
\boldsymbol{V}(q) \wedge \boldsymbol{V}(p)=\left(q_{2} p_{3}-q_{3} p_{2}\right) \boldsymbol{i}+\left(q_{1} p_{3}-q_{3} p_{1}\right) \boldsymbol{j}+\left(q_{1} p_{2}-q_{2} p_{1}\right) \boldsymbol{k}
$$


Norm of $q$ is $N_{q}=\|q\|=q \bar{q}=\bar{q} q=q_{0}{ }^{2}+q_{1}{ }^{2}+q_{2}{ }^{2}+q_{3}{ }^{2}$. If $\|q\|=1, q$ is called unit. Modulus of $q$ is $|q|=\sqrt{\|\mathrm{q}\|}$. Multiplicative inverse of non-zero real quaternion is $q^{-1}=\bar{q} /\|q\|$.

Unit real 3-dimensional sphere $S^{3}=\{q \in \mathcal{H}:|q|=1\} \subset \mathcal{H}$ constitutes a group under quaternion multiplication.

\section{Complexified Quaternion Algebra}

A complexified quaternion is in the form $Q=Q_{0}+Q_{1} \boldsymbol{i}+Q_{2} \boldsymbol{j}+Q_{3} \boldsymbol{k}$, where $\boldsymbol{i}, \boldsymbol{j}, \boldsymbol{k}$ are exactly the same as in real quaternions' obeying multiplication rules $(\boldsymbol{i}, \boldsymbol{j}, \boldsymbol{k}$ are mutually vertical unit vectors obeying the rules of multiplication $\boldsymbol{i}^{\mathbf{2}}=\boldsymbol{j}^{\mathbf{2}}=\boldsymbol{k}^{\mathbf{2}}=-\mathbf{1}$ and $\boldsymbol{i} \boldsymbol{j}=-\boldsymbol{j} \boldsymbol{i}=\boldsymbol{k}, \boldsymbol{j} \boldsymbol{k}=-\boldsymbol{k} \boldsymbol{j}=\boldsymbol{i}, \boldsymbol{k} \boldsymbol{i}=-\boldsymbol{i} \boldsymbol{k}=$ $j$ ) and $Q_{0}, Q_{1}, Q_{2}, Q_{3}$ are complex numbers (Hamilton, 1853). $Q_{0}, Q_{1}, Q_{2}$ and $Q_{3}$ are in the form $Q_{0}=$ $\mathrm{R}\left(Q_{0}\right)+\boldsymbol{I} \dot{\mathrm{i}}\left(Q_{0}\right), Q_{1}=\mathrm{R}\left(Q_{1}\right)+\boldsymbol{I} \dot{\mathrm{i}}\left(Q_{1}\right), Q_{2}=\mathrm{R}\left(Q_{2}\right)+\boldsymbol{I} \dot{\mathrm{i}}\left(Q_{2}\right), Q_{3}=\mathrm{R}\left(Q_{3}\right)+\boldsymbol{I} \dot{\mathrm{i}}\left(Q_{3}\right)$, where $\boldsymbol{I}$ is the complex imaginary operator distinct from $\boldsymbol{i}, \mathrm{R}()$ is the real part and $\mathrm{i}(\mathrm{)})$ is the imaginary part of complex number. Pure complexified quaternions form the 3-dimensional linear subspace $\operatorname{Im} \boldsymbol{H}_{\mathbb{C}}=\left\{Q_{1} \boldsymbol{i}+Q_{2} \boldsymbol{j}+\right.$ $\left.Q_{3} \boldsymbol{k}, Q_{1}, Q_{2}, Q_{3} \in \mathbb{C}\right\}$ of $\boldsymbol{H}_{\mathbb{C}}$ spanned by $\{\boldsymbol{i}, \boldsymbol{j}, \boldsymbol{k}\}$. Conjugate of $Q=Q_{0}+Q_{1} \boldsymbol{i}+Q_{2} \boldsymbol{j}+Q_{3} \boldsymbol{k}$ is $\bar{Q}=Q_{0}-$ $Q_{1} \boldsymbol{i}-Q_{2} \boldsymbol{j}-Q_{3} \boldsymbol{k}$. If $Q \in \operatorname{Im} \boldsymbol{H}_{\mathbb{C}}, \bar{Q}$ is equal to $-Q$.

Different forms can be used in order to represent a complexified quaternion (Tait, 1890; Bekar\&Yayl1, 2013). Four forms are given below:

1) $Q=\mathrm{R}(Q)+\boldsymbol{I} \dot{\mathrm{i}}(Q)=(a+\boldsymbol{\mu} b)+(c+\boldsymbol{v} d) \boldsymbol{I}$, where $\mathrm{R}(Q)=a+\boldsymbol{\mu} b$ and $\dot{\mathrm{i}}(Q)=c+\boldsymbol{v} d$ are real quaternions; $\mu, v$ are unit pure real quaternions, $\boldsymbol{\mu}^{2}=\boldsymbol{v}^{2}=I^{2}=-1$ and $a, b, c, d \in I R$. Conjugate of $Q$ is $\bar{Q}=\overline{\mathrm{R}(Q)}+\boldsymbol{I} \overline{\mathrm{i}(Q)}$.

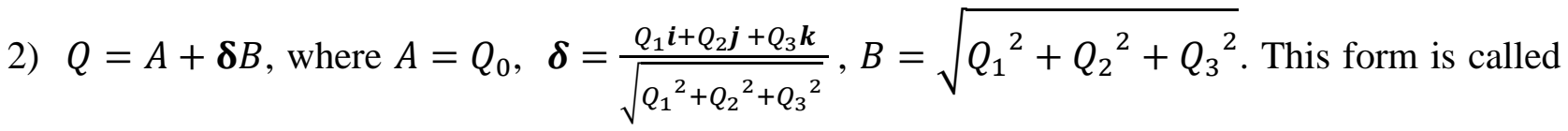
the complex form, and $\bar{Q}=A-\boldsymbol{\delta} B$.

3) $Q=S(Q)+\boldsymbol{V}(Q)$, where $S(Q)=Q_{0}$ is the scalar part and $\boldsymbol{V}(Q)=Q_{1} \boldsymbol{i}+Q_{2} \boldsymbol{j}+Q_{3} \boldsymbol{k}$ is vector part, and $\bar{Q}=S(Q)-V(Q)$.

4) $Q=\sqrt{N_{Q}}(\cos \varphi+\widehat{\boldsymbol{Q}} \sin \varphi)$, where $N_{Q}=\|Q\|={Q_{0}}^{2}+{Q_{1}}^{2}+{Q_{2}}^{2}+{Q_{3}}^{2}, \varphi \in \mathbb{C}, \quad \cos \varphi=$ $Q_{0} / \sqrt{N_{Q}}, \sin \varphi=\sqrt{{Q_{1}{ }^{2}+Q_{2}{ }^{2}+Q_{3}{ }^{2}} / \sqrt{N_{Q}}}$ and $\widehat{\boldsymbol{Q}}=\left(Q_{1} \boldsymbol{i}+Q_{2} \boldsymbol{j}+Q_{3} \boldsymbol{k}\right) / \sqrt{{Q_{1}}^{2}+Q_{2}{ }^{2}+Q_{3}{ }^{2}}$. This form is called the polar form, and $\bar{Q}=\sqrt{N_{Q}}(\cos \varphi-\widehat{\boldsymbol{Q}} \sin \varphi)$

$$
\begin{gathered}
S(Q)=a+c \boldsymbol{I}=A=Q_{0}=\sqrt{N_{Q}} \cos \varphi, \\
\boldsymbol{V}(Q)=\boldsymbol{\mu} b+\boldsymbol{v} d \boldsymbol{I}=\boldsymbol{\delta} B=\widehat{\boldsymbol{Q}} B=\sqrt{N_{Q}} \widehat{\boldsymbol{Q}} \sin \varphi,
\end{gathered}
$$

where $B=\sqrt{Q_{1}{ }^{2}+Q_{2}{ }^{2}+Q_{3}{ }^{2}}=\sqrt{N_{Q}} \sin \varphi, \boldsymbol{\delta}=\widehat{\boldsymbol{Q}}=\frac{Q_{1} \boldsymbol{i}+Q_{2} \boldsymbol{j}+Q_{3} \boldsymbol{k}}{B}$ for $B \neq 0$.

Summation of the complexified quaternions $Q$ and $P$ is $Q+P=(S(Q)+S(P))+(\boldsymbol{V}(Q)+$ $\boldsymbol{V}(P)$ ). Multiplication a complexified quaternion $Q$ with a scalar $\lambda$ is $\lambda Q=\lambda S(Q)+\lambda \boldsymbol{V}(Q)$. Multiplication of complexified quaternion $Q$ and $P$ is

$$
Q P=S(Q) S(P)-\langle\boldsymbol{V}(Q), \boldsymbol{V}(P)\rangle+S(Q) \boldsymbol{V}(P)+S(P) \boldsymbol{V}(Q)+\boldsymbol{V}(Q) \wedge \boldsymbol{V}(P),
$$

Where $\langle\boldsymbol{V}(Q), \boldsymbol{V}(P)\rangle=Q_{1} P_{1}+Q_{2} P_{2}+Q_{3} P_{3}, \boldsymbol{V}(Q) \wedge \boldsymbol{V}(P)=\left(Q_{2} P_{3}-Q_{3} P_{2}\right) \boldsymbol{i}-\left(Q_{1} P_{3}-\right.$ $\left.Q_{3} P_{1}\right) \boldsymbol{j}+\left(Q_{1} P_{2}-Q_{2} P_{1}\right) \boldsymbol{k}$. 
The norm of a complexified quaternion $Q=Q_{0}+Q_{1} \boldsymbol{i}+Q_{2} \boldsymbol{j}+Q_{3} \boldsymbol{k}$ is $N_{Q}=\|Q\|=Q \bar{Q}=$ $\bar{Q} Q={Q_{0}}^{2}+Q_{1}{ }^{2}+{Q_{2}}^{2}+{Q_{3}}^{2} \in \mathbb{C}$. If $\|Q\|=1, Q$ is a unit. $|Q|=\sqrt{\|Q\|}=\sqrt{N_{Q}}$. Multiplicative inverse of $Q$ is $Q^{-1}=\bar{Q} /\|Q\|$.

The inverse of a non-zero complexified quaternion $Q=\mathrm{R}(Q)+\boldsymbol{I} \dot{\mathrm{i}}(Q)$ is defined only when $\|\mathrm{R}(Q)\| \neq\|\mathrm{i}(Q)\|$ (i.e. $\mathrm{R}(Q) \overline{\mathrm{R}(Q)} \neq \dot{\mathrm{i}}(Q) \overline{\mathrm{i}(Q)}$ and $\mathrm{R}(Q) \overline{\mathrm{i}(Q)} \neq \dot{\mathrm{i}}(Q) \overline{\mathrm{R}(Q)}$ ). Since each non-zero complexified quaternion has an inverse, it significantly differs from real quaternions, Unit complex 3dimensional sphere $\boldsymbol{S}_{\mathbb{C}}^{3}=\left\{Q \in \operatorname{Im} \boldsymbol{H}_{\mathbb{C}}:|Q|=1\right\} \subset \operatorname{Im} \boldsymbol{\mathcal { H }}_{\mathbb{C}}$ constitutes a group under quaternion multiplication.

Any complexified quaternion $Q=Q_{0}+Q_{1} \boldsymbol{i}+Q_{2} \boldsymbol{j}+Q_{3} \boldsymbol{k}$ can be written as $Q=\left(Q_{0}+Q_{1} \boldsymbol{i}\right)+$ $\left(Q_{2}+Q_{3} \boldsymbol{i}\right) \boldsymbol{j}=T+S \boldsymbol{j}$. Thus, the vector space $\boldsymbol{H}_{\mathbb{C}}$ onto $\mathbb{C}_{2}$ is 2-dimensional, where $T=Q_{0}+Q_{1} \boldsymbol{i}, S=$ $Q_{2}+Q_{3} i$ satisfying $I i=i I$.

Right multiplication by $Q=T+S \boldsymbol{j}$ is equal to left multiplication by the matrix

$$
\mathbf{A}=\left[\begin{array}{rr}
T & -\bar{S} \\
S & \bar{T}
\end{array}\right]
$$

If we take $Q$ as unit (i.e. restricting complexified quaternion to unit complex 3-sphere $\boldsymbol{S}_{\mathbb{C}}^{3}$ ) we get $\|Q\|=\|S\|+\|T\|=1$, that means $\mathbf{A}$ is a unique complex unitary matrix. These matrices are composed of the group of unique complex unitary $2 \times 2$ matrices $\boldsymbol{S} \boldsymbol{U}_{\mathbb{C}}(2)$.

$\psi: \boldsymbol{S}_{\mathbb{C}}^{3} \rightarrow \boldsymbol{S} \boldsymbol{U}_{\mathbb{C}}(2)$ is one-to-one and onto not quite an isomorphism. However, it satisfies

$$
\psi(Q P)=\psi(P) \psi(Q) \text { for } P \in \boldsymbol{S}_{\mathbb{C}}^{3}
$$

\section{RESULTS AND DISCUSSION}

\section{Parallels of Latitude and Meridians of Longitude on $S U_{\mathbb{C}}(2)$}

Corresponding $\boldsymbol{S} \boldsymbol{U}_{\mathbb{C}}(2)$ to $\boldsymbol{S}_{\mathbb{C}}^{3}$ helps us to utilize spherical concepts on $\boldsymbol{S} \boldsymbol{U}_{\mathbb{C}}(2)$. Let $Q=Q_{0}+$ $Q_{1} \boldsymbol{i}+Q_{2} \boldsymbol{j}+Q_{3} \boldsymbol{k}=\left(Q_{0}+Q_{1} \boldsymbol{i}\right)+\left(Q_{2}+Q_{3} \boldsymbol{i}\right) \boldsymbol{j}=T+S \boldsymbol{j}$ be a complexified quaternion, then

$$
\mathbf{A}(T, S)=\left[\begin{array}{rr}
T & -\bar{S} \\
S & \bar{T}
\end{array}\right],|T|^{2}+|S|^{2}=1,
$$

The characteristic polynomial for $\mathbf{A}(T, S)$ is

$$
\begin{gathered}
|\mathbf{A}(T, S)-t I|=(T-t)(\bar{T}-t)+S \bar{S} \\
=t^{2}-(T+\bar{T}) t+1 \\
=t^{2}-2 \mathrm{R}(T) t+1 .
\end{gathered}
$$

As for fixed $Q_{0} \in[-1,+1]$, the $\mathrm{R}(T)=Q_{0} \in \mathbb{C}$ ranges from -1 to +1 , we accept $\left\{\mathbf{A}(T, S): \operatorname{R}(T)=Q_{0}, Q_{0} \in \mathbb{C}\right\}$ the parallel of latitude at $Q_{0}$. It is seen that when their characteristic polynomials are the same, two unique complex unitary matrices take place on the identical parallel of latitude. The latitude parallels that correspond to $Q_{0}=1$ and $Q_{0}=-1$ are the sole-point sets $\{I\}$ and $\{-I\}$, corresponding, respectively, to north and south poles. For $-1<Q_{0}<1$, the parallel of latitude at $Q_{0}$ is topologically a 2-complex sphere that sits in $\boldsymbol{S}_{\mathbb{C}}^{3}$. This is obvious by calculating

$$
|T|^{2}+|S|^{2}=1 \text {, and } \mathrm{R}(T)=Q_{0}
$$

in complex coordinates and geometrically as well, because the parallel of latitude at $Q_{0}$ is equivalent to the slice cut out from $\boldsymbol{S}_{\mathbb{C}}^{3}$ by the 3-dimensional complex space defined by $\mathrm{R}(T)=Q_{0}$ in $\mathbb{C}_{2}^{2}$. Because $\mathrm{R}(T)=1 / 2$ trace $\mathbf{A}(T, S)$, the equator $Q_{0}=0$ represent to traceless matrices. The longitude meridians are gigantic circles which go throughout the poles. One significant longitude meridian is given by the diagonal matrices in $\boldsymbol{S} \boldsymbol{U}_{\mathbb{C}}(2)$. For a diagonal $\mathbf{A}(T, S)$ we have $Q_{0}=0$, thus $|T|^{2}=1$. Letting $T=e^{i \varphi}$, 
where $\boldsymbol{i}$ is the complex imaginary operator of bicomplex number T, a diagonal matrix can be shown as follows:

$$
\left[\begin{array}{cc}
e^{i \varphi} & 0 \\
0 & e^{-i \varphi}
\end{array}\right] \in \boldsymbol{S U}_{\mathbb{C}}(2), \quad \varphi \in \mathbb{C}
$$

These longitude meridians cut the equator at

$$
\left[\begin{array}{cc}
\boldsymbol{i} & 0 \\
0 & -\boldsymbol{i}
\end{array}\right]
$$

and its negative.

Remark. The conjugacy element of $\mathbf{A} \in \boldsymbol{S} \boldsymbol{U}_{\mathbb{C}}(2)$ is the set $\left\{\boldsymbol{C A C}^{-1}: \mathbf{C} \in \boldsymbol{S} \boldsymbol{U}_{\mathbb{C}}(2)\right\}$.

Because $\operatorname{Tr}\left(\boldsymbol{C} \mathbf{A C}^{-1}\right)=\operatorname{Tr} \mathbf{A}$, a parallel of latitude contains conjugacy component. It is easy to show that the opposite is saund as well, hence the parallels of latitude are absolutely the conjugacy elements of matrices in $\boldsymbol{S} \boldsymbol{U}_{\mathbb{C}}(2)$.

The depiction of $\boldsymbol{S} \boldsymbol{U}_{\mathbb{C}}(2)=\boldsymbol{S}_{\mathbb{C}}^{3}$ according to the parallels of latitude does not offer new things concerning the geometry of $\boldsymbol{S}_{\mathbb{C}}^{3}$. Therefore, the identical geometric picture is also sound in the which has lower number of dimension of $\boldsymbol{S}_{\mathbb{C}}^{2}$.

\section{Complex-Clifford Tori}

The unit complex 3-sphere $\boldsymbol{S}_{\mathbb{C}}^{3}$ can be parameterize by two bicomplex variables $(T, S)$ which satisfy $|T|^{2}+|S|^{2}=1$. It should be noted that $T$ runs on the first factor and $S$ on the second one of $\mathbb{C}_{2}^{2}=\mathbb{C}_{2} \times$ $\mathbb{C}_{2}$. Let us take the function

$h: \boldsymbol{S}_{\mathbb{C}}^{3} \rightarrow \mathbb{C}, \quad h(T, S)=|T|^{2}-|S|^{2},(T, S) \in \boldsymbol{S}_{\mathbb{C}}^{3}$.

Since $|T|^{2}+|S|^{2}=1$, we have $-1 \leq h \leq 1$. We aim to envision the level sets $C_{Q_{0}}=\left\{(T, S) \in S_{\mathbb{C}}^{3}: h(T, S)=Q_{0}\right\},-1<Q_{0}<1$.

We have $(T, S) \in C_{Q_{0}}$ if $|T|^{2}-|S|^{2}=Q_{0}$, and since $|T|^{2}+|S|^{2}=1$, we get and, since $|T|^{2}+$ $|S|^{2}=1$, adding and substracting yields

$$
|T|^{2}=\frac{1+Q_{0}}{2},|S|^{2}=\frac{1-Q_{0}}{2} .
$$

For $Q_{0}=1$. It is obtained $|T|=1, S=0 . C_{1}$ is the unit circle taking place in the first factor of $\mathbb{C}_{2}^{2}=\mathbb{C}_{2} \times \mathbb{C}_{2}$. Similar to this, $C_{-1}$ is the unit circle taking place in the second factor of $\mathbb{C}_{2}^{2}=\mathbb{C}_{2} \times \mathbb{C}_{2}$. Especially, $C_{1}$ and $C_{-1}$ are vertical. When we assume $-1<Q_{0}<1$, we understand that the right-hand parts of the equations above are positive. Those parts are uncoupled, which means that the first refers to a circle about the origin with radius $\sqrt{\left(1+Q_{0}\right) / 2}$ in the first factor of $\mathbb{C}_{2}^{2}$ and the second refers to a resembling circle with $\sqrt{\left(1-Q_{0}\right) / 2}$ radius in the second factor of $\mathbb{C}_{2}^{2}$. Consequently,

$$
C_{Q_{0}}=\left\{(T, S) \in \boldsymbol{S}_{\mathbb{C}}^{2}:|T|^{2}=\frac{1+Q_{0}}{2},|S|^{2}=\frac{1-Q_{0}}{2}\right\}
$$

is the the complexified quaternion obtained the Cartesian product of two complex circles - a complex torus. Apart from $C_{\mp 1}$, the $C_{Q_{0}},-1<Q_{0}<1$ decompose $\boldsymbol{S}_{\mathbb{C}}^{3}$. Visually it can be interpretted as: Imagine ourselves in $\boldsymbol{S}_{\mathbb{C}}^{3}$ motion along the gigantic circle $C_{-1}=\left\{\left(0, e^{i \varphi}\right): \varphi \in \mathbb{C}\right\}$. The direction that we are moving is seen at each point. We realize that we are surrounded by a 3-dimensional complex space as we are in $\boldsymbol{S}_{\mathbb{C}}^{3}$. When we go around $C_{-1}$ and drag the complex circle along, in addition to keeping it vertical to our path, it will sweep a complex-Clifford torus. If we increase the radius of the circle which we transport, we take fatter tori. At the other marginal value, which is $Q_{0}=1$, the tori decrease to $C_{1}$.

If the quaternion imaginary parts (i.e. $\dot{\mathrm{i}}(Q)$ for $Q=\mathrm{R}(Q)+\boldsymbol{I} \dot{\mathrm{i}}(Q) \in \mathcal{H}_{\mathbb{C}}$ ) of unit complexified quaternions and unit complex numbers are assumed to be zero, we get $\boldsymbol{S} \boldsymbol{U}_{\mathbb{C}}(2)=\boldsymbol{S}^{3}$. So, $C_{Q_{0}}$ is a torus 
obtained by the the cartesian product of two circles. In this declaration, tori $C_{Q_{0}}$ are called Clifford tori, where $Q_{0} \in[-1,1]$ (Ata\&Yayl, 2009). In the example given below, Flat torus in $\boldsymbol{S}^{3}$, will be given which is one of the most amazing surfaces in 4-dimensional space (Fig. 1).

Example. The flat torus is formed by

$$
(a, b) \rightarrow(\cos (a+b), \sin (a+b), \cos (a-b), \sin (a-b),
$$

where $0 \leq a \leq 2 \pi, 0 \leq b \leq 2 \pi$. Summing these four coordinates' squares equals to 1 , thus that the flat torus is entirely comprised in $\boldsymbol{S}^{3}$. This torus is coverlaid by circles, and projecting stereographically from the pole $(0,0,0,1)$ on the 3 -sphere $\boldsymbol{S}^{3}$ shows that the image in $\boldsymbol{S}^{3}$ is a flat torus of revolution overlaid by circles and every circle goes around the torus once in each direction. Any two circles among them are linked in that a disc limited by any of them is cut precisely once by another one (Hamilton, 1853).

If we remove the middle complex flat torus $C_{0}$ from $\boldsymbol{S}_{\mathbb{C}}^{3}$, it is seen that $\boldsymbol{S}_{\mathbb{C}}^{3}$ is divided into the discrete union of two solid tori. Therefore, it is possible to note that the $\boldsymbol{S}_{\mathbb{C}}^{3}$ is got from two solid tori by pasting them together with their boundaries.

Imagine $\boldsymbol{S}_{\mathbb{C}}^{1}=\left\{e^{i \varphi}: \varphi \in \mathbb{C}\right\} \subset \mathbb{C}_{2}$ acting on $\boldsymbol{S}_{\mathbb{C}}^{3}$ by the 4-complex dimensiomal complex rotation $e^{i \varphi}(T, S) \rightarrow\left(e^{i \varphi} T, e^{i \varphi} S\right)$ (this refers to left complex quaternionic multiplication of unit complexified quaternion by $e^{i \varphi} \in \boldsymbol{S}_{\mathbb{C}}^{1}$ ). Each orbit represents a gigantic complex circle and each one is comprised in a Complex-Clifford tori. Actually, the orbit

$$
\boldsymbol{S}_{\mathbb{C}}^{1}\left(T_{0}, S_{0}\right)=\left\{\left(e^{i \varphi} T_{0}, e^{i \varphi} S_{0}\right): \varphi \in \mathbb{C}\right\}
$$

is the intersection of $\boldsymbol{S}_{\mathbb{C}}^{3}$ with the 2-complex dimensional linear subspaces in $\mathbb{C}_{2}^{2}$ satisfying $T S_{0}-S T_{0}=$ 0 . As

$$
\begin{aligned}
h\left(e^{i \varphi} T, e^{i \varphi} S\right) & =\left|e^{i \varphi} T\right|^{2}-\left|e^{i \varphi} S\right|^{2} \\
& =|T|^{2}-|S|^{2} \\
& =h(T, S),
\end{aligned}
$$

the second statement also follows.

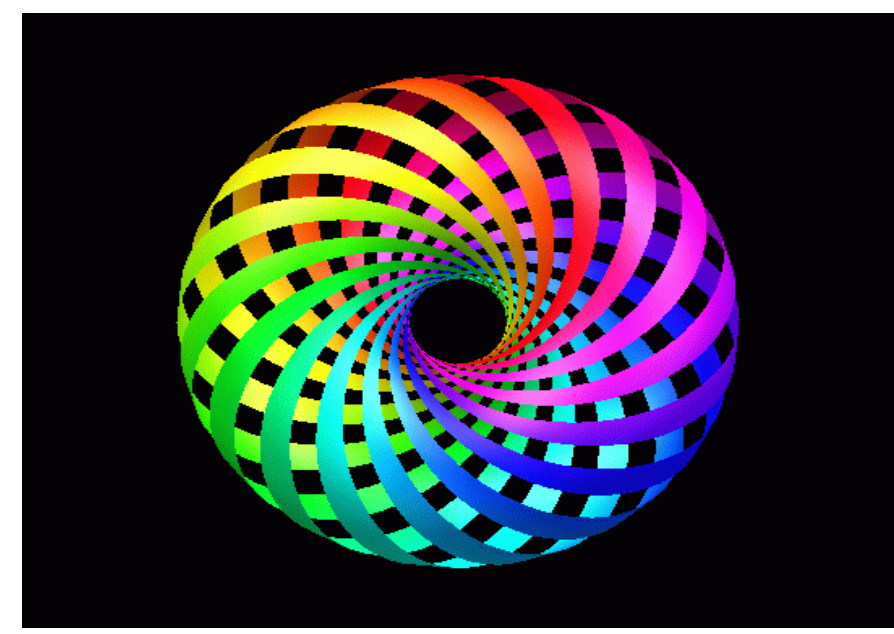

Figure.1. Flat torus 
Theorem. The groups $\boldsymbol{S}_{\mathbb{C}}^{3} / \boldsymbol{S}_{\mathbb{C}}^{1}$ and $\boldsymbol{S}_{\mathbb{C}}^{3}$ are homeomorphic.

Proof. The way to associate an orbit $\boldsymbol{S}_{\mathbb{C}}^{1}\left(T_{0}, S_{0}\right)=\left\{\left(e^{i \varphi} T_{0}, e^{i \varphi} S_{0}\right): \varphi \in \mathbb{C}\right\}$, a unique point on $\boldsymbol{S}_{\mathbb{C}}^{2}$ is to identify the projection mapping $\boldsymbol{S}_{\mathbb{C}}^{3} \rightarrow \boldsymbol{S}_{\mathbb{C}}^{3} / \boldsymbol{S}_{\mathbb{C}}^{1}$, where $\left|T_{0}\right|^{2}+\left|S_{0}\right|^{2}=1$. Hopf fibriation is a mapping

defined by

$$
F: S_{\mathbb{C}}^{3} \rightarrow S_{\mathbb{C}}^{2}
$$

$$
\left.F(T, S)=\left(|T|^{2}-|S|^{2}\right), 2 T \bar{S}\right) \in \mathbb{C x} \mathbb{C}_{2}=\mathbb{C}^{3}
$$

Since

$$
|F(T, S)|^{2}=\left(|T|^{2}-|S|\right)^{2}+4|T|^{2}|S|^{2}=\left(|T|^{2}+|S|^{2}\right)^{2}=1
$$

if $(T, S) \in \boldsymbol{S}_{\mathbb{C}}^{3}$. Secondly, $F$ is constant under the action of $\boldsymbol{S}_{\mathbb{C}}^{1}$, because

$$
\begin{aligned}
F\left(e^{i \varphi} T, e^{i \varphi} S\right) & =\left(\left|e^{i \varphi} T\right|^{2}-\left|e^{i \varphi} S\right|^{2}, 2 e^{i \varphi} S\right) \\
& =\left(|T|^{2}-|S|^{2}, 2 T \bar{S}\right) \\
& =F(T, S) .
\end{aligned}
$$

Therefore, $F$ maps every orbit of $\boldsymbol{S}_{\mathbb{C}}^{1}$ in $\boldsymbol{S}_{\mathbb{C}}^{3}$ into a sole complex point. In order to reveal that $\boldsymbol{S}_{\mathbb{C}}^{3} / \boldsymbol{S}_{\mathbb{C}}^{1}=\boldsymbol{S}_{\mathbb{C}}^{3}$ , it is necessary to demonstrate the fact that the orbits represent the inverse images of complex points of $\boldsymbol{S}_{\mathbb{C}}^{2}$. That means, we need to whenever $F\left(T_{1}, S_{1}\right)=F\left(T_{2}, S_{2}\right)$, the points $\left(T_{1}, S_{1}\right)$ and $\left(T_{2}, S_{2}\right)$, are in the same orbit under $\boldsymbol{S}_{\mathbb{C}}^{1}$. Since Hopf fibriation images which is translated into

$$
\left|T_{1}\right|^{2}-|S|^{2}=\left|T_{2}\right|^{2}-\left|S_{2}\right|^{2} \text { and } T_{1} \overline{S_{1}}=T_{2} \overline{S_{2}} \text {, }
$$

the first equality shows that $\left(T_{1}, S_{1}\right)$ and $\left(T_{2}, S_{2}\right)$ are on the identical Complex-Clifford torus $C_{Q_{0}}$. Therefore we get

$$
\left|T_{1}\right|^{2}=|T|^{2}=\frac{1+Q_{0}}{2}, \quad\left|S_{1}\right|^{2}=\left|S_{2}\right|^{2}=\frac{1-Q_{0}}{2} .
$$

Taking $T_{2}=e^{i \varphi} T_{1}$ and $S_{2}=e^{i \beta} S_{1}$, we get

$$
T_{1} \overline{S_{1}}=e^{i(\varphi-\beta)} T_{1} \overline{S_{1}}
$$

Thus (excluding the trivial cases when $T_{1}=0$ or $S_{1}=0$, which might be handled separately)

$$
e^{i(\varphi-\beta)}=1
$$

follows. Based on the periodicity feature of the complex exponential function, $\varphi, \beta$ are the same. Thus, $S_{2}=e^{i \beta} S_{1}$.

Looking at two orbits of the action of $\boldsymbol{S}_{\mathbb{C}}^{1}$ on $\boldsymbol{S}_{\mathbb{C}}^{3}$ (on the identical complex-Clifford torus), reveals that they are "joined" in $\boldsymbol{S}_{\mathbb{C}}^{3}$. This shows that the Hopf fibriation $F: \boldsymbol{S}_{\mathbb{C}}^{3} \rightarrow \boldsymbol{S}_{\mathbb{C}}^{2}$ can not be distorted constantly throughout maps into a ceaseless map $\boldsymbol{S}_{\mathbb{C}}^{3} \rightarrow \boldsymbol{S}_{\mathbb{C}}^{2}$ which transmit $\boldsymbol{S}_{\mathbb{C}}^{3}$ to a sole complex point. That means that $F$ is homotopically nontrivial.

\section{CONCLUSION}

Spherical concepts like parallels of latitude and meridians of longitude on $\boldsymbol{S} \boldsymbol{U}_{\mathbb{C}}(2)$ are pointed out by utilizing the one-to-one comparable segment of $\boldsymbol{S}_{\mathbb{C}}^{3}$ and $\boldsymbol{S} \boldsymbol{U}_{\mathbb{C}}(2)$. Homology group of complex 2sphere $\boldsymbol{S}_{\mathbb{C}}^{2}$ is zero with respect to Hopf fibriation, it is revealed that the third homology group $\boldsymbol{S}_{\mathbb{C}}^{2}$ also becomes zero when we use Hopf fibriation. Flat torus in $\boldsymbol{S}^{3}$ is given as an example for Complex-Clifford tori. To do this, quaternion imaginary parts taken of unit complexified quaternion and unit complex numbers are accepted as zero. 


\section{ACKNOWLEDGEMENTS}

The authors would like to thank referees for their careful reading and their valuable suggestions and comments that helped us to improve the presentation of this paper.

\section{REFERENCES}

Ata E, Yayli Y, 2009. Dual quaternions and dual projective spaces. Chaos, Solitons \& Fractals, 40(3), 1255-1263.

Bekar M, Yayli Y, 2013. Involutions of complexified quaternions and split quaternions. Advances in Applied Clifford Algebras, 23(2), 283-299.

Chevalley C, 1946. Theory of Lie groups Princeton Univ. Press, Princeton, NJ-USA.

Hamilton W R, 1844. On a new species of imaginary quantities connected with a theory of quaternions. In Proceedings of the Royal Irish Academy (Vol. 2, No. 424-434, pp. 4-1).

Hamilton W R, 1853. Chapter VI in: Lectures on Quaternions. Hodges and Smith, Dublin, Available online at Cornell University Library: http://historical.library.cornell.edu/math/. (Date of access: 16 June 2019).

Tait P G, 1890. An elementary treatise on quaternions. University Press, Michigan-USA.

Toth G, 1998. Glimpses of algebra and geometry. Springer Science \& Business Media, NY-USA. 